\title{
RELATIONS ENTRE LE VOLUME DE LA CARCASSE DES BOVINS ET LE POIDS DE SES DIFFÉRENTS COMPOSANTS ANATOMIQUES OU CHIMIQUES
}

\author{
J. ROBELIN \\ avec la collaboration technique de \\ Colette Raynal-Rongìri, R. Jailler et G. CUYLle \\ Laboratoive de la Production de Viande, \\ Centre de Recherches de Clermont-Ferrand, I. N. R. A., \\ Theix, Saint Genès Champanelle, 63110 Beaumont
}

RÉSUMÉ

Nous avons calculé la relation entre le volume de la carcasse de 3 I jeunes bovins mâles de 3 races, et le poids des différents composants anatomiques (squclette, muscles, clépôts adipeux) ou chimiques (eau, matières grasses, minéraux, protéines) de cette carcasse. La relation est très étroite : le coefficient de corrélation multiple est égal à 0,999 .

A même poids de carcasse, le volume est lié en premier lieu au poids des dépôts adipeux $(\mathrm{R}=0,44)$ ou des lipides $(\mathrm{R}=0,42)$, mais à même état d'cngraissement il est lié également a la composition de la masse maigre (proportion de muscles ou de matières minérales et de protéines). Compte tenu de la variabilité de la composition de la masse maigre entre races, nous pensons que la relation entre le volume et l'état d'engraissement des carcasses serait plus étroite à l'intérieur de chaque race. In calcul effectué à partir de nos premiers résultats tend à confirmer cette hypothèse.

\section{INTRODUCTION}

Parmi les nombreuses méthodes permettant d'estimer la composition anatomique ou chimique de la carcasse des bovins, la méthode densimétrique a retenu l'attention de nombreux chercheurs (cf. Revue de Pearson et al., ig68). A la suite des travaux de BeHnke (I94I) sur l'Homme, et de ceux de Rathbun et PACE (I945) sur le Cobaye, des études ont été entreprises sur les Porcins (BROWn et al., I95I ; DESMOULIN, I970), les Ovins (Kirton et Barton, I958; Meyer, ig62; Timon et Bichard, i965; Khandekar et al., I965; Pradhan et al., I966; RAtTray et al., I973), et les Bovins (Kraybili, et al., I952; Garret et Hinman, I969; Gil et al., I970; Ledger et 
Gilliver, I973; PRESTON et al., I974). Ces différents travaux ont montré que la densité des carcasses ou du corps entier était surtout reliée à la proportion de dépôts adipeux ou de matières grasses. Cependant, les études entreprises sur les bovins ont porté sur des animaux de race Anglo-saxonne, dont l'état d'engraissement était très important.

Nous nous sommes demandé si cette méthode densimétrique était applicable à des animaux ayant un état d'engraissement plus faible, et en particulier si elle pouvait être utilisable dans le cas des taurillons de races françaises.

Nous nous sommes surtout attaché aux aspects méthodologiques et analytiques de la méthode, peu abordés jusqu'à présent : précision de la mesure du volume d'une carcasse, relation entre le volume et la répartition pondérale des différents composants de la carcasse. L'étude de ces deux aspects paraît en effet nécessaire, avant de calculer des équations d'estimation de la proportion des différents composants à partir du volume et du poids de la carcasse. Cette analyse a été réalisée à partir de 3I carcasses de jeunes bovins mâles entiers abattus entre 9 et I6 mois (tab1. I), disséquées afin d'interpréter des expériences nutritionnelles.

TABLE I

Caractéristiques des animaux

\begin{tabular}{|c|c|c|c|c|}
\hline Races & Frisonne & Charolaise & Limousine & $\begin{array}{l}\text { Ensemble } \\
\text { des races }\end{array}$ \\
\hline Nombre d'animaux & 11 & 8 & 12 & 31 \\
\hline $\begin{array}{l}\text { Carcasse }(\mathrm{kg} \pm \mathrm{SD})\left({ }^{1}\right) \ldots \ldots \\
\text { Volume }\left(\mathrm{dm}^{\mathrm{a}} \pm \mathrm{SD}\right) \ldots \ldots \ldots\end{array}$ & $\begin{array}{l}236,1 \pm 60,3 \\
218,4 \pm 56,5\end{array}$ & $\begin{array}{l}316,3 \pm 69,5 \\
294,3 \pm 65,7\end{array}$ & $\begin{array}{l}282,0 \pm 76,3 \\
261,5 \pm 72,1\end{array}$ & $\begin{array}{l}274,6 \pm 74,2 \\
254,7 \pm 70,0\end{array}$ \\
\hline 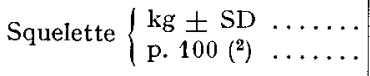 & $38,3 \underset{16,2}{ \pm} 7,7$ & $\begin{array}{c}42,2 \pm 7,0 \\
13,3\end{array}$ & $\begin{array}{c}35,6 \pm 7,1 \\
12,6\end{array}$ & $\begin{array}{c}38,2 \pm 7,5 \\
13,9\end{array}$ \\
\hline Muscles $\left\{\begin{array}{l}\mathrm{kg} \pm \mathrm{SD} \ldots \ldots \\
\mathrm{p} .100 \ldots \ldots\end{array}\right.$ & $\begin{array}{c}158,5 \pm 39,7 \\
67,1\end{array}$ & $\underset{72,4}{229,0 \pm 51,1}$ & $\underset{74,8}{211,0 \pm 54,4}$ & $\begin{array}{c}197,0 \pm 55,8 \\
71,7\end{array}$ \\
\hline $\begin{array}{c}\text { Dépôts } \\
\text { adipeux }\end{array}\left\{\begin{array}{l}\mathrm{kg} \pm \mathrm{SD} \ldots \ldots \\
\mathrm{p} .100 \ldots \ldots\end{array}\right.$ & $\begin{array}{c}37,1 \pm 14,1 \\
15,7\end{array}$ & $\begin{array}{c}4_{ \pm} 2,4 \pm 12,8 \\
13,4\end{array}$ & $\begin{array}{c}33,2 \pm 15,4 \\
11,8\end{array}$ & $\begin{array}{c}37,0 \pm 14,3 \\
13,5\end{array}$ \\
\hline Eau $\left\{\begin{array}{l}\mathrm{kg} \pm \mathrm{SD} \ldots \ldots \\
\mathrm{p} .100 \ldots \ldots\end{array}\right.$ & $\underset{60,5}{142,8 \pm 32,5}$ & $\begin{array}{c}199,1 \frac{1}{1} 40,5 \\
62,9\end{array}$ & $\begin{array}{c}180,8 \pm \underset{64,1}{4} \\
45,8\end{array}$ & $\begin{array}{c}172,0 \pm 45,2 \\
62,6\end{array}$ \\
\hline $\begin{array}{c}\text { Matières } \\
\text { grasses }\end{array}\left\{\begin{array}{l}\mathrm{kg} \pm \mathrm{SD} \ldots \ldots \\
\mathrm{p} .100 \ldots \ldots\end{array}\right.$ & $\begin{array}{c}33,7 \pm 14,0 \\
14,3\end{array}$ & $\begin{array}{c}40,0 \pm 13,7 \\
12,6\end{array}$ & $\begin{array}{c}29,8 \pm 12,8 \\
10,6\end{array}$ & $\begin{array}{c}33,9 \pm 13,6 \\
12,3\end{array}$ \\
\hline $\begin{array}{c}\text { Matières } \\
\text { minérales }\end{array}\left\{\begin{array}{l}\mathrm{kg} \pm \mathrm{SD} \ldots \ldots \\
\mathrm{p} .100 \ldots \ldots\end{array}\right.$ & $13,2 \underset{5,6}{ \pm} 3,2$ & $14,4 \pm \underset{4,5}{ \pm} 2,8$ & $13,3 \underset{4,7}{ \pm} 3,0$ & $13,6 \underset{4,9}{ \pm} 3,0$ \\
\hline Matières $\left\{\begin{array}{l}\mathrm{kg} \pm \mathrm{SD} \ldots \ldots \\
\text { azotées } 100 \ldots \ldots\end{array}\right.$ & $\begin{array}{c}14,1 \pm 1 \\
18,7\end{array}$ & $\begin{array}{c}60,2 \pm 13,7 \\
19,0\end{array}$ & $55,9 \pm 15,0$ & $\operatorname{sen}_{19,2} \pm 14,6$ \\
\hline
\end{tabular}

(1) Poids en $\mathrm{kg}(\mathrm{SD}=$ écart-type en $\mathrm{kg}$ ).

(2) Poids exprimé en pourcentage du poids de la carcasse. 


\section{MATÉRIEL E'T MÉTHODES}

\section{Mesure de la composition anatomique ct chimique de la carcasse}

Le poids des différents tissus (squelette, muscles, dépôts adipeux) a été mesuré après dissection de la demi-carcasse droite. Ainsi que nous l'avons déjà décrit (RoBelin, GeAy et BERANGER, 1974), ces tissus ont ensuite été broyés et analysés séparément : mesure de la teneur en eau, lipides, minéraux, protéines et énergie.

Nous avons déterminé le poids des différents tissus et composants chimiques de la carcasse chaude entière, à partir de la composition de la demi-carcasse disséquée. Nous avons admis que la perte de poids entre l'abattage et la dissection, perte due à l'évaporation, était proportionnelle au poids de chacun des tissus.

\section{Détermination du volume de la carcasse}

Nous avons mesuré le volume de la demi-carcasse gauche, après avoir retiré la queue ct les piliers latéraux et médians du diaphragme (" hampe " et "onglet ").

Le volume de la demi-carcasse gauche a été calculé par différence entre le poids $P$ (peséc dans l'air) et le poids $P i$ de la demi-carcasse mesuré après immersion dans l'eau. Le poids $P$ a été mesuré, à Ioo $\mathrm{g}$ près, immédiatement après l'abattage. Le poids $P i$ a été mesuré après une durée de ressuyage de 18 à 24 heures en chambre réfrigérée à $+4^{\circ} \mathrm{C}$, à l'aide d'une balance dont la précision était de $5 \mathrm{~g}$. La demi-carcasse coupée en deux quartiers, a été immergée dans une cuve cylindrique (hauteur : $200 \mathrm{~cm}$, diamètre : $140 \mathrm{~cm}$ ), contenant de l'eau dont la température était comprise entrc $\mathrm{I}^{\circ} \mathrm{C}$ et $\mathrm{I} 3^{\circ} \mathrm{C}$. Les variations de cette température restant faibles, nous n'en avons pas tenu compte pour calculer le volume de la carcasse. De plus, la masse spécifique de l'eau à $\mathrm{I} 2^{\circ} \mathrm{C}$ étant peu différente de I $(0,999$ 97), nous avons calculé le volume de la carcasse dircctement par différence entre $\mathrm{P}$ et $\mathrm{P} i$.

\section{Etude mathématique des résultats}

Soit $\mathrm{P}, \mathrm{V}, \mathrm{X}_{1}, \mathrm{X}_{2}, \mathrm{X}_{3}$ respectivement le poids de la carcasse, son volume et le poids de ses différents composants anatomiques. Nous voulons savoir si $\mathrm{P}$ et $\mathrm{V}$ permettent d'estimer lc poids $\mathrm{X}_{1}$ (dépôts adipeux). Nous savons tout d'abord qu'il existe théoriquement deux relations mathématiques entre ces différentes variables :

$$
\begin{aligned}
& \text { I }: P=X_{1}+X_{2}+X_{3} \\
& 2: V=\alpha_{1} X_{1}+\alpha_{2} X_{2}+\alpha_{3} X_{3}
\end{aligned}
$$

La somme des poids des composants est égale au poids de la carcasse, la somme de leurs volumes $\left(\alpha_{1}, \alpha_{2}, \alpha_{3}\right.$ étant les volumes massiques des composants) est égale au volume de la carcasse.

En réalité, $\mathrm{V}, \mathrm{X}_{1}, \mathrm{X}_{2}$ et $\mathrm{X}_{3}$ sont des valeurs mesurées avec une erreur expérimentale. La relation 2 est donc une relation statistique que nous pouvons établir entre $\mathrm{V}$ et $\mathrm{X}_{1}, \mathrm{X}_{2}, \mathrm{X}_{3}$ :

$$
3: V=\beta_{1} x_{1}+\beta_{2} x_{2}+\beta_{3} x_{3}+\beta_{4}
$$

$\beta_{1}, \beta_{2}, \beta_{3}$ coefficients de $X_{1}, X_{2}, X_{3}$ sont alors des estimations des volumes massiques $\alpha_{1}, \alpha_{2}, \alpha_{3}$.

Pour que $P$ et $V$ permettent d'estimer $X_{1}$ par exemple, il faut en premier lieu que $V$ soit étroitement relié à $\mathrm{X}_{1}, \mathrm{X}_{2}, \mathrm{X}_{3}$, c'est-à-dire que la variance résiduelle de la relation 3 soit faible.

Néanmoins, cette condition n'est pas suffisante pour que $X_{1}$ soit lié à $V$ et $P$. Il faut en plus que la variabilité de $\mathrm{V}$ soit liée en priorité, sinon en totalité, à la variabilité de $\mathrm{X}_{1}$. La mise en évidence de cette dernière liaison n'est pas possible à partir de l'équation 3 , car $V, X_{1}, X_{2}, X_{3}$ sont liés au poids de la carcasse $P$; nous avons donc tout d'abord éliminé de l'équation 3 l'influence du poids de la carcasse; pour ce faire, nous avons calculé l'équation de régression de chacune des variables $\mathrm{V}, \mathrm{X}_{1}, \mathrm{X}_{2}, \mathrm{X}_{3}$ avec $\mathbf{P}$ :

$$
\begin{aligned}
& 4: \mathrm{V}=a \mathrm{P}+b \\
& 5: \mathrm{X}_{1}=a_{1} \mathrm{P}+b_{1} \\
& 6: \mathrm{X}_{2}=a_{2} \mathrm{P}+b_{2} \\
& 7: \mathrm{X}_{3}=a_{3} \mathrm{P}+b_{3}
\end{aligned}
$$


Ensuite, pour chacune des carcasses, nous avons calculé les écarts résiduels aux droites de régression $4,5,6$ ct 7 , respectivement $v, v_{1}, v_{2}, v_{3}$, qui sont par définition des variables indépendantes de P. Par exemple, pour la carcasse $i$, dont le poids cst P $i$, le volume Vi, l'écart résiduel à la régression 3 est égal à :

$$
v i=\mathrm{V} i-(a \mathrm{P} i+b)
$$

Enfin, nous avons étudié la relation 8 :

$$
8: v=g\left(x_{1}, x_{2}, x_{3}\right)
$$

Par régression progressive, nous avons pu faire la part des différents composants dans la variabilité du volume de la carcasse, indépendamment du poids de carcasse.

\section{RÉSULTATS ET DISCUSSION}

\section{I. - Précision de la mesure du volume de la carcasse.}

Afin d'estimer la répétabilité de la mesure de $\mathrm{P} i$, nous avons effectué sur I7 demi-carcasses, trois pesées successives en retirant la demi-carcasse de l'eau entre chacune d'elles. Le poids $\mathbf{P} i$ (voisin de $\mathrm{Io} \mathrm{kg}$ ), a augmenté de $35 \mathrm{~g}$ en moyenne ( $\pm \mathrm{I} 5 \mathrm{~g}$ ) entre la première et la seconde pesée, de $7,5 \mathrm{~g}$ seulement $( \pm 5 \mathrm{~g})$ entre la seconde et la troisième pesée. Compte tenu des poids moyens $\mathbf{P}$ que nous avons enregistrés ( $\mathrm{r}_{5} \mathrm{O} \mathrm{kg}$ environ), l'erreur relative sur le calcul dı volume à partir d'une seule pesée est égale à 2,I p. I 000 . Il est probable que les variations du poids $\mathrm{P} i$ sont dues à des bulles d'air qui restent sur les parois de la carcasse après immersion; ce phénomène a déjà été signalé par KIRTON et BARTON (I958).

Le volume réel de la carcasse, et par conséquent la mesure qu'on peut en faire, dépendent de la durée écoulée entre l'abattage et la mesure. Nous avons observé, sur 2 demi-carcasses, une diminution rapide au cours des premières 24 heures (I0,5 p. I OOo du volume initial); cette diminution se ralentit par la suite I,67 p. I ooo entre 24 et $4^{8}$ heures, 0,89 p. I ooo entre $4^{8}$ et 72 heures (mesures effectuées sur 6 demi-carcasses). Au cours des nombreux travaux réalisés jusqu'à présent sur la densité des carcasses d'animaux, la mesure $\mathrm{P} i$ a été effectuée en des temps variables après l'abattage selon les auteurs, de I 8 heures (KIRTON et BARTON, I958; GIL, et al., I970) à 7 jours (KHANDEKAR et al., r965) sans que ces auteurs, sauf KIINE et al. (I955), insistent particulièrement sur la standardisation de cette durée, qui nous parait importante.

Compte tenu de cette évolution de $\mathrm{P} i$ avec le temps, et de l'air qui reste sur les parois des carcasses après immersion, l'erreur expérimentale sur le volume, dans les conditions où nous l'avons mesuré (une seule pesée I 8 à 24 heures après 1'abattage) serait voisine de 3 p. I ooo. Les conditions de la mesure du poids $\mathrm{P} i$ sont donc beaucoup plus importantes à prendre en compte que la précision de la balance. En particulier, il paraît inutile d'effectuer cette pesée au gramme près, comme le font la plupart des auteurs dont nous avons cité les travaux; une précision de $5 \mathrm{~g}$ est suffisante dans le cas des bovins.

Les volumes massiques des deux côtés d'une même carcasse, sans queue ni piliers du diaphragme, ne sont pas significativement différents ; la différence moyenne obtenue sur 8 comparaisons est égale à $2, \mathrm{I} \mathrm{dm} / \mathrm{g}$, avec un écart-type de $\mathrm{I}, 3 \mathrm{dm}^{3} / \mathrm{g}$. Quelle que soit sa valeur, cette différence a peu d'importance. En revanche, sa varia- 
bilité entraîne une erreur relative supplémentaire sur l'estimation du volume de la carcasse totale égale à $2,4 \mathrm{p}$. I ooo.

$\mathrm{Si}$, comme nous l'avons fait pour des raisons pratiques, on établit des relations entre le volume de la carcasse mesuré sur une demi-carcasse, et la composition anatomique et chimique mesurée sur l'autre demi-carcasse, l'erreur relative totale sur la mesure du volume est donc égale à $3+2,4$, soit $5,4 \mathrm{p}$. I 000 , soit $\mathrm{I}, 400 \mathrm{dm}^{3}$ en moyenne pour les carcasses que nous avons utilisées dont le volume moyen était de $260 \mathrm{dm}^{3}$.

\section{2. - Infuence de la composition anatomique ou chimique de la carcasse sur les variations de son volume}

Nous avons obtenu la relation suivante entre le volume $\mathrm{V}\left(\mathrm{dm}^{3}\right)$ d'une carcasse et le poids $(\mathrm{kg})$ des différents composants anatomiques, squelette (S), muscles (M), dépôts adipeux (D) :

$$
\begin{aligned}
& \mathrm{V}=0,86 \mathrm{~S}+0,94 \mathrm{M}+\mathrm{I}, 02 \mathrm{D}-2, \mathrm{I} 7 \\
& \mathrm{~N}=3 \mathrm{I} \quad \mathrm{R}^{2}=0,99^{8} \quad \mathrm{~S}=0,90 \quad \text { (cf. équation 3) }
\end{aligned}
$$

$\mathrm{N}$ est le nombre d'observations ayant permis de calculer l'équation, $\mathrm{R}^{2}$ le carré du coefficient de corrélation multiple entre $V$ et $S, M, D, S D$ l'écart-type résiduel de la régression exprimé en $\mathrm{dm}^{3}$.

Entre le volume $\mathrm{V}$, et le poids $(\mathrm{kg})$ des composants chimiques, eau $(\mathrm{E})$, matières grasses $(G)$, matières minérales $(M)$ et protéines $(P)$, la relation obtenue est la suivante :

$$
\begin{aligned}
& \mathrm{V}=0,95 \mathrm{E}+\mathrm{I}, \mathrm{I} 3 \mathrm{G}+0,54 \mathrm{M}+0,90 \mathrm{P}-\mathrm{I}, 36 \\
& \mathrm{~N}=3 \mathbf{I} \quad \mathrm{R}^{2}=0,999 \quad \mathrm{SD}=0,83 \quad \text { (cf. équation 3) }
\end{aligned}
$$

D'après ces deux relations, la composition anatomique ou chimique de la carcasse explique plus de 99 p. Ioo de la variabilité de son volume. Les erreurs de pesée des différents composants de la carcasse et l'erreur due à la variabilité de leur volume massique seraient égales à $0,4 \mathrm{dm}^{3}$ seulement. La variabilité résiduelle étant très faible, le volume massique des différents composants anatomiques ou chimiques est donc certainement peu variable entre carcasses.

Les coefficients des relations citées plus haut sont théoriquement des estimateurs de ces volumes massiques (cf. équation 3). Ces relations font intervenir un terme constant (respectivement 2,17 et $I, 36$ ) ; mais, il n'est pas significativement $(P<0,0 I$ ) différent de 0 . Ces estimations des volumes massiques sont relativement voisines de celles qui ont été calculées par différents auteurs (KRAYBILL et al., I952 ; LOFGREEN et Garreit, I954; BIEBER et SAFFLE, I96I).

Les équations précédentes permettent d'étudier globalement l'influence de la composition de la carcasse sur les variations de son volume, mais elles ne nous permettent pas de faire la part respective de chacun des composants. En effet, les coeffcients de corrélation simples (tabl. 2) entre le volume et le poids de chacun des composants ont une signification très limitée, dans la mesure où le poids des différents composants sont reliés au poids de la carcasse (tabl. 3) et donc liés entre eux.

Pour éliminer une partie de ces liaisons, et en particulier celle qui est due au poids de la carcasse, nous avons étudié la relation entre le volume et le poids des 
TABLEAU 2

Coefficients de corrélation entre le poids des différents composants et le volume de la carcasse

\begin{tabular}{|c|c|c|c|}
\hline $\begin{array}{l}\text { Composants } \\
\text { anatomiques }\end{array}$ & $\mathbf{R}$ & $\begin{array}{c}\text { Composants } \\
\text { chimiques }\end{array}$ & $\mathrm{R}$ \\
\hline $\begin{array}{l}\text { Muscles } \\
\text { Dépòts adipeux } \\
\text { Squelette }\end{array}$ & $\begin{array}{l}0,98 \\
0,87 \\
0,84\end{array}$ & $\begin{array}{l}\text { Eau } \\
\text { Matières grasses } \\
\text { Protéines } \\
\text { Matières minérales }\end{array}$ & $\begin{array}{l}0,99 \\
0,86 \\
0,99 \\
0,91\end{array}$ \\
\hline
\end{tabular}

TABLEAU 3

Coefficients de corrélation entre les différents composants de la carcasse des bovins

A. - Composants anatomiques

\begin{tabular}{l|c|c|c|c}
\hline & $\begin{array}{c}\text { Poids } \\
\text { de la carcasse }\end{array}$ & $\begin{array}{c}\text { Poids } \\
\text { des muscles }\end{array}$ & $\begin{array}{c}\text { Poids } \\
\text { du squelette }\end{array}$ \\
\hline dépóts adipeux
\end{tabular}

B. - Composants chimiques

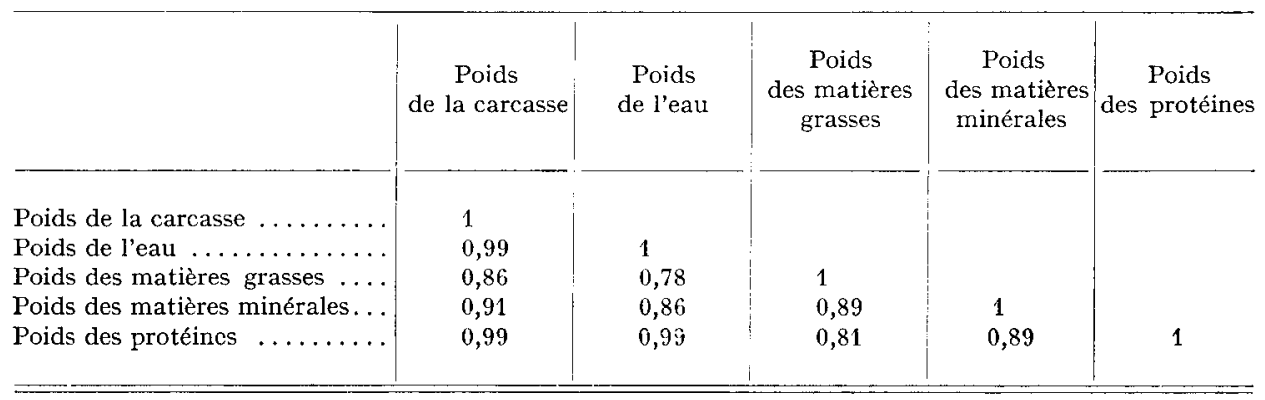


composants à poids de carcasse égal (cf. § matériel et méthodes). Dans ces conditions, le volume est lié en premier lieu au poids des dépôts adipeux $(R=0,44)$ ou des matières grasses $(\mathrm{R}=0,42)$ (tab1. 4).

TABLEAU 4

Résultats de l'analyse de régression progressive à poids de carcasse constant entre le volume de la carcasse et le poids des différents composants anatomiques ou chimiques de la carcasse

\begin{tabular}{|c|c|c|c|c|c|c|}
\hline \multirow{5}{*}{$\begin{array}{l}\text { Palier } n^{0} 1 \\
\text { Palier } n^{0} 2 \\
\text { Palier } n^{\circ} 3\end{array}$} & \multicolumn{4}{|c|}{ Variables introduites } & \multirow{2}{*}{$F^{*}(\mathbf{1})$} & \multirow{2}{*}{$R\left({ }^{(2)}\right)$} \\
\hline & 1 & 2 & 3 & 4 & & \\
\hline & Dépôts adipeux & & & & $* *$ & $0, t^{\prime} t^{\prime}$ \\
\hline & Dépôts adipeux & Muscles & & & $* *$ & 0,64 \\
\hline & Dépôts adipeux & Muscles & Squelette & & NS & 0,65 \\
\hline Palier $n^{\circ} 1$ & Matières grasses & & & & ** & 0,42 \\
\hline Palier $n^{\circ} 2$ & Matières grasses & Matières minérales & & & $* *$ & 0,74 \\
\hline Palier no 3 & Matières grasses & Matières minérales & Protéines & & NS & 0,74 \\
\hline Palier $n^{\circ} 4$ & Matières grasses & Matières minérales & Protéines & Eau & NS & 0,75 \\
\hline
\end{tabular}

(1) Test de signification de la nouvelle variable introduite à chaque palier :

** $=\mathrm{P}<0,01$

$\mathrm{NS}=$ non significatif.

( $\left.{ }^{2}\right)$ Coefficient de corrélation multiple (ou simple au palier $n^{\circ} 1$ ).

Mais, à poids de dépôts adipeux ou de matières grasses donné, les variations du volume sont également liées au poids des muscles ou des matières minérales $(\mathrm{P}<\mathrm{O}, \mathrm{OI})$.

Ces variations du volume correspondent donc à des variations dans la composition de la masse maigre à poids de carcasse et à état d'engraissement constants ; d'ailleurs, un calcul portant sur le petit nombre d'animaux dont nous disposions nous a montré qu'effectivement la race avait une influence significative $(P<0,0 I)$ sur la relation entre le volume et l'état d'engraissement des carcasses. De plus, lorsqu'on retire l'effet de la race, le volume de la carcasse est indépendant de la composition de la masse maigre.

\section{3. - Estimation de la composition de la carcasse des bovins $\grave{a}$ partir du poids et du volume de la carcasse}

Le nombre d'animaux dont nous disposons dans chaque race ne nous permet pas de proposer des équations de régression généralisables, permettant d'estimer l'état d'engraissement des carcasses à partir de leur poids et de leur volume. Néanmoins, nous avons rapporté au tableau 5 les premières équations que nous avons calculées à partir de l'ensemble des animaux (toutes races confondues). Nous constatons tout d'abord, qu'après le poids de carcasse, le volume n'apporte une infor- 
mation significative $(\mathrm{P}<\mathrm{o}, \mathrm{OI})$ que dans l'estimation du poids des matières grasses ou des dépôts adipeux. L'écart-type résiduel de la régression, voisin de $6,5 \mathrm{~kg}$ pour un poids de carcasse moyen de $275 \mathrm{~kg}$, est comparable aux résultats de KRAYBII, et al. (1952), Garrett (I968), Garrett et Hinman (I969), Gil ct al. (I970), Lediger et Gilitiver (I973) ou de PrEston et al. (1974). Cependant, cet écart-type est légèrement supérieur à celui qu'on obtient avec l'estimation à partir de la II côte (GEAy et Beranger, I969).

\section{TABLEAU 5}

Estimation du poids des différents composants $(\mathrm{X})$ de la carcasse des bovins $\grave{a}$ partir du poids (P) et du volume (V) de la carcasse

$$
\left(\mathrm{X}=b_{1} \mathrm{P}+b_{2} \mathrm{~V}+c\right)\left({ }^{\mathbf{1}}\right)
$$

\begin{tabular}{|c|c|c|c|c|c|c|}
\hline Composants & $b_{1}$ & $b_{2}$ & $c$ & $\mathrm{R}\left({ }^{2}\right)$ & $S\left({ }^{3}\right)$ & $F(4)$ \\
\hline $\begin{array}{l}\text { Squelette } \ldots \ldots \ldots \ldots \\
\text { Muscles . . . . . . } \\
\text { Dépôts adipeux } \ldots \ldots\end{array}$ & $\begin{array}{r}0,76 \\
2,91 \\
-2,88\end{array}$ & $\begin{array}{r}0,71 \\
-2,30 \\
3,23\end{array}$ & $\begin{array}{r}11,53 \\
-16,28 \\
5,38\end{array}$ & $\begin{array}{l}0,8499 \\
0,9853 \\
0,8911\end{array}$ & $\begin{array}{l}4,11 \\
9,86 \\
6,73\end{array}$ & $\begin{array}{l}0,88 \\
1,57 \\
6,73^{* *}\end{array}$ \\
\hline $\begin{array}{l}\text { Fau ................... } \\
\text { Matières grasses. ....... } \\
\text { Matières minérales } \ldots \ldots \\
\text { Matières azotées } \ldots \ldots \ldots\end{array}$ & $\begin{array}{r}2,36 \\
-2,66 \\
0,39 \\
0,69\end{array}$ & $\begin{array}{r}-1,86 \\
2,98 \\
-0,38 \\
-0,53\end{array}$ & $\begin{array}{r}1,67 \\
3,61 \\
1,79 \\
-\quad 3,09\end{array}$ & $\begin{array}{l}0,9901 \\
0,8852 \\
0,9211 \\
0,9932\end{array}$ & $\begin{array}{l}6,55 \\
6,56 \\
1,21 \\
1,76\end{array}$ & $\begin{array}{l}2,32 \\
6,02 * * \\
2,84 \\
2,65\end{array}$ \\
\hline 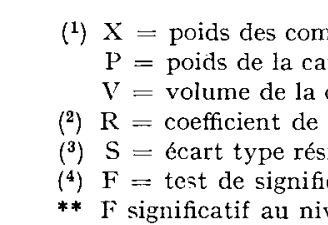 & $\begin{array}{l}\text { osants en } \\
\text { casse en kq } \\
\text { arcasse en } \\
\text { orrélation } \\
\text { Auel de la } \\
\text { ation de l' } \\
\text { eau } 0,01 \mathrm{~s}\end{array}$ & $\begin{array}{l}{ }^{3} \\
\text { altiple entr } \\
\text { ression }(\mathrm{kg} \\
\text { port de la } \\
\text { est supérie }\end{array}$ & $\begin{array}{l}Y \text { et }\left(X_{1}, X\right. \\
\text { conde varia } \\
\text { à } 4,04 \text {. }\end{array}$ & $X_{2}$ & & \\
\hline
\end{tabular}

L'analyse de ces premiers résultats confirme que le volume de la carcasse dépend en premier lieu du poids des dépôts adipeux ou des matières grasses, à poids de carcasse fixé ; mais elle montre également que le volume dépend de la composition de la masse maigre (rapport muscle/os, en particulier) variable entre races.

Nous devons donc poursuivre cette étude avec un plus grand nombre d'animaux de chaque race, de façon à proposer des équations d'estimation adaptées à chacune d'entre elles.

Reçu pour publication en février 1975.

\section{REMERCIEMENTS}

Nous tenons à remercier R. Tomassone (Laboratoire de Biométrie du C. N. R. Z. de Jouy en Josas) pour sa contribution à l'analyse statistique des résultats. 


\title{
SUMMARY
}

\author{
REI,ATIONSHIP BETWEEN THE VOLUME OF THE BEEF CARCASS \\ AND THE WEIGHT OF ITS ANATOMICAL AND CHEMICAL COMPONENTS
}

Thirty one young bulls of 3 breeds were used to calculate the relationship between the volume of the carcass and the weight of its different anatomical components (skeleton muscles, fat depots) or chemical components (water, fat, minerals, protein). There was a very close relationship, the multiple correlation coefficient being 0.999 .

At the same carcass weight, the volume was principally related to the weight of fat depots $(\mathrm{R}=0.44)$ or lipids $(\mathrm{R}=0.42)$, but at the same weight of fat, it was also related to the composition of the lean mass (proportion of muscles or minerals and protein). On account of the variability between breeds as regards the composition of the lean mass, we assume that the relationship between the volume and the degree of fatness of the carcass would be closer within each breed. A calculation made from our first findings tends to confirm this hypothesis.

\section{RÉFÉRENCES BIBLIOGRAPHIQUES}

JЗенNкL, I94I. Cité par BRozeck J., KEY A., I95I. The evaluation of thickness-fatness in man : norms and interrelationships. Brit. J. Nutr., 5, 194-202.

BrEBer 1). D., SAFFLE R. I., I96r. Calculation of fat and protein content of beef from specific gravity and moisture. J. Anim. Sci., 20, 239-243.

Brows C. J., Hiller J. C., Whatley J. A., i95I. Specific gravity as a measure of the fat content of the pork carcass. J. Anim. Sci, 10, 97-103.

Desmoritar B., г97o. La détermination de la clensité corporelle. I. Principes et conditions d'une mesure directe de l'état d'engraissement de la carcasse de porc. Journées Réch. porcine en France, I7I-I75, I. N. R. A., I. T. I'. éd, Paris.

Garrett W. N., I968. Experiences in the use of body density as an estimator of body composition of animals. In Body composition of Animals and Man. Pub. 1598. National Acadeny of Sciences Washingrton, I). C.

Garrett W. N., Hixiax $N$., 1969. Re-evaluation of the relationship between carcass density and body composition of beef steers. J. Anim. Sci., 28, I-5.

Geay Y., Beranger C., 1969. Estimation de la composition de la carcasse de jeunes bovins à partir de la composition d'un morceau monocostal prélevé au niveau de la I I côte. Ann. Zootech., 18, 65-77.

Gil E. A., Johnson R. R., Cahill V. R., McClere K. J., Klosterman E. W., ig7o. An evaluation of carcass specific volume, dye dilution, and empty body parameters as predictors of beef carcass composition over a wide range of fatness. J. Anim. Sci., 31, 459-469.

Khandekar V. N., MeNanus W. R., Goldstoni: C. L., r965. Some indices of carcass composition of dorset horn top-cross lambs. II. Specific gravity as an index of the fat content of the carcass and various joints. J. Agr. Sci., 65, I55-I 58 .

Kirton A. H., Barton R. A., i958. Specific gravity as an index of fat content of mutton carcasses and various joints. N. Z.J.A gric. Res. 1,633-64I.

Kline li. A., Asirton G. C., Kastelic J., I955. The effect of chilling time on the specific gravity of hog carcass and upon the correlation between specific gravity and measures of fatness. J.Anim. Sci., 14, 1 230 .

Kraybill H. F., I3itter Il. L., Hankins O. G., 1952. Body composition of cattle. II. Determination of fat and water content from measurement of body specific gravity. J. Appl. Physiol., 4, 575-583.

Ledger H. P., Gilliver B., I973. An examination of sample joint dissection and specific gravity techniques for assessing the carcass composition of steers slaughtered in commercial abattoirs. J. A gric. Sci, 80, 381-392.

I ofgreen G. P., Garrett W. N., I954. Creatine excretion and specific gravity as related to the composition of the 9-IO-I th rib cut of Hereford steers. J. Anim. Sci., 13, 496-499.

Merer J. H., ig62. Removing sources of error in lamb feeding experiments. J.Anim. Sci, 21, I27-131.

Pearson A. M., Purchas R. W., Reineke E. P., ig68. Theory and potential usefulness of body density as a predictor of body composition. In Body composition of Animals and Mans. Pub. I598. National Academy of Sciences Washington. D. C. 
Pradhan S. I., McManus W. R., Coldstone C. I., Hart R. F., lihandekar V. N., Arnold (i. W., I966. Indices of the rarcass composition of Dorset Horn top-cross lambs. III. Relationships between chemical composition, specific gravity and weight of carcass and joints. J. Agric. Sci., 66, 4I-47.

Prestox R. L., Vance R. D., Cahill V. R., Kock S. W., I974. Carcass specific gravity and carcass composition in cattle and the effect of bone proportionality on this relationship. J. Anim. Sci., 38, $47-5 \mathrm{I}$.

Rathbun E. N., Pace N., I945. Studies on body composition. I. The determination of total body fat by means of the body specific gravity. J. Biol. Chem., 158, 667-676.

Rattray R. V., Garrett W. N., Hinman N., East N. H., Meyer H. H., ig73. Relationships between carcass density and body composition with observations on differences in the fat free body composition in sheep. J. Anim. Sci., 37, $1332-\mathrm{r} 338$.

Rometin J., Gray Y., Beranger C., I975. Estimation de la composition chimique des carcasses de jeunes bovins mâles à partir de la proportion de dépôts adipenx d'un morceau monocostal prélevé au niveau de la i ${ }^{\mathbf{e}}$ côte. Ann. Zootech., 24, 325-326.

Timon V. M., Bichard M., I965. Quantitative estimates of lamb carcass composition. III. Carcass measurements and a comparison of the predictive efficiency of sample joint composition, carcass specific gravity determinations and carcass measurements. Anim. Prod., \%, 189-201. 